Service social

\title{
Le virage ambulatoire et le service social en milieu de santé. Réflexions et propositions
}

Volume 45, numéro 3, 1996

Santé

URI : https://id.erudit.org/iderudit/706744ar

DOI : https://doi.org/10.7202/706744ar

Aller au sommaire du numéro

Éditeur(s)

École de service social de l'Université Laval

ISSN

1708-1734 (numérique)

Découvrir la revue

Citer cet article

(1996). Le virage ambulatoire et le service social en milieu de santé. Réflexions et propositions. Service social, 45(3), 175-191. https://doi.org/10.7202/706744ar d'utilisation que vous pouvez consulter en ligne.

https://apropos.erudit.org/fr/usagers/politique-dutilisation/ 


\section{COMMENTAIRES ET DOCUMENTS}

\section{Le virage ambulatoire et le service social en milieu de santé Réflexions et propositions*}

L'ASSOCIATION DES PRATICIENS DE SERVICE SOCIAL EN MILIEU DE SANTÉ DU QUÉBEC

Le présent document se veut l'occasion, pour l'Association des praticiens de service social en milieu de santé, dans le cadre de la reconfiguration actuelle du réseau de la santé et des services sociaux, de situer sa contribution en tant qu'acteur concerné. Elle saisit, par la même occasion, l'opportunité de repenser la conception des services au-delà de la logique contemporaine de la production des soins.

De nombreuses forces de changement, de même que bon nombre de dynamiques sociales, font que les services de santé

* Ce document, paru en 1996, a été réalisé par :

Louise Gagnon-Hotte, Centre hospitalier universitaire de l'Estrie; Huguette Ouellet, Régie régionale, santé et services sociaux de Québec; Gilles Poirier, Centre hospitalier régional de l'Outaouais ; Johanne St-Denis, Centre hospitalier Charles-LeMoyne, Montérégie ; Régina Thibodeau, Centre hospitalier CharlesLeMoyne, Montérégie ; Lise Tousignant, Hôpital Jeffery Hale, Québec. 
sont en profonde mutation. L'Organisation mondiale de la santé, les normes canadiennes de l'agrément des établissements de santé et l'article 81 de la Loi sur les services de santé et les services sociaux donnent au service social certaines assises dans le domaine de la santé. La politique québécoise de la santé et du bien-être est aussi venue marquer un tournant décisif, en dépassant largement le cadre conceptuel de l'approche médicale ou biomédicale. Elle réoriente les services dans une optique de prévention et de promotion face aux problèmes sociaux et de santé.

Marc Renaud ${ }^{1}$, dont les recommandations ont inspiré la politique santé et bien-être, se dit convaincu que "quelque chose » d'autre contribue à influer sur la santé. Il situe clairement ce «quelque chose» dans l'environnement social, économique et culturel des gens. Pour lui, l'élément le plus important à considérer dans le cadre de la santé serait celui de l'environnement social. Céline Mercier, dans la revue Les Diplômés du printemps 1993, rapporte les écrits de Marc Renaud: "Ce que l'on commence à intuitionner, c'est ce que certains des déterminants les plus fondamentaux de la santé ont quelque chose à voir avec le sentiment de contrôle sur sa vie (empowerment) et la capacité de faire face aux difficultés (coping skills) qui eux-mêmes sont liés à la postérité d'un pays, aux expériences de l'enfance, au soutien social et à bien d'autres facteurs. »

Le service social hospitalier, dans une telle perspective, se voit confirmé dans sa mission et sa raison d'être et se sent particulièrement interpellé dans le présent cadre d'organisation, que ce soit au plan des soins ambulatoires ou encore de l'approche horizontale des programmes-clientèles, pour réaffirmer ses rôles et fonctions auprès des clientèles. Le défi sera de contribuer à améliorer la santé de la population en influençant certains éléments de l'environnement social des personnes qui transiteront dans les milieux hospitaliers.

Le présent document sur le service social hospitalier et le virage ambulatoire précise l'impact et les manifestations de celuici sur l'hôpital en devenir. Il identifie la clientèle de l'hôpital et particulièrement celle du service social. Sont ensuite regardées les répercussions possibles sur la clientèle et les adaptations que celle-ci sera appelée à faire, de même que l'apport du service social et les défis que cette reconfiguration l'amène à relever.

1. Marc Renaud est sociologue de la santé à l'Université de Montréal, vice-président de l'Institut canadien de la santé et président du Conseil québécois de la recherche sociale. 


\section{LE VIRAGE AMBULATOIRE}

Bien qu'aucune définition spécifique du virage ambulatoire n'ait fait l'objet de consensus, ce concept renvoie à un ensemble de réalités sous-tendu par une même orientation, ayant cependant des manifestations et des impacts différents selon l'angle d'où il est regardé.

Le Petit Robert donne des définitions de virage et de ambulatoire: virage signifie «changement important d'orientation» et ambulatoire, "capable de se déplacer».

En associant l'un et l'autre de ces deux mots et en les adaptant au système de santé, virage ambulatoire peut se définir comme un changement important de direction du système de santé québécois orienté vers des personnes pouvant se déplacer, soit que la personne se déplace pour aller vers le service sans y être admise, soit que le service se déplace pour aller vers la personne. De façon plus spécifique, ce virage signifie le passage de l'utilisation de services institutionnalisés à des services dispensés de façon prépondérante près du milieu de vie de la personne avec des infrastructures plus légères.

Ce concept a ses assises, ses impacts sur un hôpital en devenir, ses manifestations particulières pour les milieux hospitaliers.

\section{Ses assises}

Le virage ambulatoire s'appuie sur plusieurs éléments qui ont changé la dynamique du système de santé. Sont considérés les besoins changeants de la population (vieillissement, incidences de la pauvreté...), l'évolution des technologies et des pratiques dans les services de santé, telles que la modification des traitements, des médications et les mesures augmentant la sécurité à domicile. Les orientations actuelles axent le système de santé vers le maintien de la personne dans la communauté, la consolidation des services préventifs et des services de base, la complémentarité, la continuité et la coordination de la gamme de services reliés à la santé, de même que vers la modification des modes de pratique de tous les professionnels concernés.

S'ajoutent les changements dans les comportements de consommation des clientèles impliquant des niveaux d'exigence de plus en plus élevés, une volonté de prise en charge de leur santé et de participation active aux décisions les concernant, 
une demande d'informations claires et précises afin de donner un consentement éclairé aux services et aux soins, une exploration des solutions alternatives aux traitements médicaux.

Un autre élément non négligeable, souvent perçu à tort comme le seul fondement du changement d'orientations du système de santé, touche la rationalisation des coûts axée sur l'efficience et sur l'efficacité des interventions pour améliorer la santé et le bien-être de la population. La tendance actuelle de croissance des coûts de la santé n'entraîne pas une amélioration proportionnelle de l'état de santé, d'où un nécessaire requestionnement sur l'efficacité des interventions posées pour apporter des solutions durables aux clientèles.

\section{Ses impacts sur l'hôpital en devenir}

Cette nouvelle orientation aura des impacts majeurs sur les milieux hospitaliers dans toutes leurs composantes. Son devenir est à définir avec les différents acteurs.

Ce milieu aussi est en évolution : il sera différent de son passé et de son présent. Déjà plusieurs impacts sont prévisibles, dont:

- l'hôpital devient un élément de la gamme de services et non l'élément principal, d'où passage d'un système centré sur l'hôpital comme dispensateur privilégié des soins de santé à un système orienté vers la dispensation de soins dans le milieu;

- l'hôpital devra être en interaction avec la communauté et les services qui y sont requis et offerts;

- l'hôpital mettra à contribution les expertises spécifiques et particulières de chaque catégorie de professionnels, de ses services de pointe et de son infrastructure;

- l'hôpital établira un équilibre entre le développement de hautes technologies, des interventions de pointe et l'efficience de ces technologies en relation avec les ressources investies par rapport aux autres déterminants de la santé;

- l'hôpital recevra des clientèles diversifiées et différentes : moins de personnes requérant des services courants et de base consulteront en centre hospitalier;

- l'hôpital modifiera sa façon de dispenser les soins et services, les lieux de distribution et adaptera son mode organisationnel, dont la révision de l'efficacité et de la pertinence 
de certaines interventions, l'offre de services spécialisés en clinique externe ou autres en remplacement de l'hospitalisation, la consultation auprès des partenaires.

Déjà, la plupart des milieux hospitaliers se mobilisent pour prendre ou intensifier ce virage ambulatoire. Les manifestations sont et seront de différents ordres :

Un passage vers des services moins institutionnalisés et institutionnalisants

- programme d'évaluation préadmission et préintervention;

- chirurgie de jour ;

- admission le jour de l'intervention;

- diminution de la durée moyenne de séjour;

- planification de congé dès la préadmission;

- unité de court séjour $(24,48,72$ heures) avec congé prédéterminé ;

- service de médecine de jour;

- service de consultations externes et de soins alternatifs ;

- utilisation nécessaire des services courants (CLSC, cliniques privées);

- modification des lieux de dispensation de services;

- rationalisation des lits de courte durée;

- service d'enseignement aux clientèles.

Un accent sur la dispensation de services nécessitant la structure plus spécialisée et plus «lourde» des centres hospitaliers

- mise à contribution des expertises spécialisées et surspécialisées: "l'hôpital offrira les 10 à $15 \%$ des services spécialisés et les rendra souvent à l'extérieur» (Rochon, 1995);

- utilisation efficiente et efficace des ressources hospitalières ;

- pratique des soins et services liés aux spécialités médicales de niveau régional et suprarégional, les services de base et les services courants étant offerts et accessibles dans des structures plus légères et possiblement plus près des clientèles;

- service aux clientèles nécessitant une masse critique et des plateaux techniques plus structurés. 


\section{Une concentration de clientèles diversifiées et différentes}

- une clientèle vieillissante ;

- une clientèle vivant des maladies évolutives et chroniques ;

- une clientèle présentant une combinaison de problématiques ou pathologies;

- une clientèle vivant de nouvelles problématiques;

- une clientèle présentant des problèmes «rares» ou moins répandus dans la population ;

- des clientèles vulnérables.

Ce changement de cap oblige chacun des acteurs touchés, de la clientèle aux dispensateurs de soins et services de tous les niveaux, à des adaptations et à de nouvelles stratégies d'utilisation des forces respectives. À titre de dispensateurs de services, ce changement demande de recadrer l'action et de la recentrer sur ce qui est pertinent (service qui améliorera le plus l'état de santé et dont les avantages dépassent suffisamment les risques), efficace (service qui améliore l'état de santé), efficient (service fourni là où il peut être le plus rentable au moment où l'efficacité est optimale), nécessaire et souhaité par le client.

\section{LES CLIENTÈLES}

La plupart du temps, un séjour plus ou moins prolongé en centre hospitalier, avec ce que cela comporte comme diminution des capacités physiques, traitements exigeants, souffrance physique, place les personnes dans un état important de vulnérabilité et de détresse. Cependant, la bonne majorité d'entre elles possèdent des ressources personnelles et le soutien de leur milieu nécessaires pour composer avec la maladie et faire face aux conséquences ou ajustements qu'elle suppose.

Certaines, toutefois, nécessitent une aide particulière et un soutien supplémentaire pour retrouver leur équilibre, le maintenir, pour s'adapter aux conséquences que la maladie apporte ou pour procéder aux changements qu'elle entraîne.

Le rôle du service social hospitalier se situe au carrefour des liens qui existent entre la maladie et le fonctionnement social des personnes ou entre les conditions sociales et l'état de santé des individus. 


\section{La clientèle-cible}

De façon générale, une aide spécifique est particulièrement nécessaire dans les situations suivantes:

- le diagnostic posé est sévère, critique ou réservé ;

- la situation familiale ou sociale de la personne est complexe et précaire ;

- la personne présente un certain degré de vulnérabilité de par sa condition ou ses capacités personnelles;

- la personne est en besoin de protection sociale.

\section{Les principales caractéristiques de la clientèle-cible}

Que ce soit dans les départements spécialisés et de soins généraux, en externe ou à l'interne, dans le but de dépister les clientèles à risque, de procéder à une évaluation ou d'apporter un traitement psychosocial, toute personne présentant des indices de vulnérabilité ou des caractéristiques de difficultés psychosociales devrait faire l'objet d'une référence au service social.

De façon plus particulière, sont visées les personnes

- présentant un profil gériatrique;

- seules, isolées et sans support social;

- ayant connu des hospitalisations à répétition ;

- présentant une hygiène négligée ou de la malnutrition;

- dont la situation sociale ou familiale est perturbée ;

- dont les proches vivent de l'épuisement;

- victimes d'abus, de négligence ou de violence;

- ayant une grossesse difficile ou à risque ;

- parents de bébés naissants prématurés, handicapés ou mort-nés ;

- déjà connues des services de maintien à domicile avant l'hospitalisation ;

- qui nécessitent des services de maintien à domicile après l'hospitalisation ;

- dont le pronostic de durée de vie est réservé ;

- aux prises avec une maladie grave, chronique ou dégénérative ; 
- ayant subi un traumatisme important ;

- qui ont de la difficulté à composer avec la maladie ou qui présentent des signes de détresse;

- dont la situation ou la condition pose des questions éthiques ;

- dont la situation ou la condition pose des questions éthiques.

\section{Le virage ambulatoire et la clientèle-cible}

Le virage ambulatoire, sans changer l'aperçu général de l'ensemble de la clientèle décrite, viendra sûrement en modifier le profil. En effet, dans la perspective où les personnes retrouveront dans leur milieu de vie la majorité des réponses à leurs problèmes de santé et dans la perspective où les durées de séjour seront ramenées au minimum, il est facile d'entrevoir que la clientèle hospitalisée, qui sera dirigée vers le service social, sera la plus lourde au point de vue de l'état de santé et de l'autonomie fonctionnelle, présentera un haut degré de complexité au plan de la dynamique et de la problématique ou sera la plus vulnérable sur les plans affectif, familial et social.

La clientèle hospitalisée étant plus lourde et séjournant moins longtemps, il est prévisible que le virage ambulatoire aura des répercussions sur les activités du service social. De fait, la diminution des durées de séjour et la rationalisation des lits de courte durée auront pour effet d'augmenter le taux de roulement pour chacun des lits des centres hospitaliers et, par voie de conséquence, d'accroître le nombre potentiel de références au service social ou, à tout le moins, la nécessité d'une intervention concentrée.

\section{La concertation et la transmission des connaissances}

Pour réaliser ses objectifs, le virage ambulatoire commande une organisation de soins et services en «réseau » pour les clientèles. Il implique la contribution de plusieurs acteurs et une nécessaire concertation.

Ces nouveaux acteurs constituent un autre type de «clients» avec lesquels le service social est appelé à travailler, dans un but de collaboration, de partage d'expertise et d'enrichissement réciproque.

Les «clients » seront plus particulièrement les collègues des autres disciplines à l'intérieur du centre hospitalier, ceux des 
autres établissements du réseau, les partenaires du secteur privé ou communautaire, les associés ponctuels ou plus réguliers des milieux scolaires, juridiques, municipaux, etc.

Ainsi, les connaissances que les services sociaux hospitaliers ont développées et acquises en ce qui a trait aux impacts psychosociaux de la maladie et à l'intervention psychosociale spécialisée dans le domaine de la santé pourront être communiquées à ces collaborateurs et partagées avec eux.

En retour, ces derniers communiqueront leur savoir et leurs compétences dans leur domaine d'expertise, démontrant de cette manière un souci commun de concertation et de services de qualité aux clientèles desservies.

\section{L'APPORT DU SERVICE SOCIAL HOSPITALIER AU VIRAGE AMBULATOIRE}

\section{L'interface entre les aspects sociaux et biologiques}

"On sait depuis longtemps que l'état de santé d'une population est le résultat dynamique de l'évolution et de l'interaction de quatre grandes catégories de facteurs: les facteurs biologiques, les habitudes de vie, les conditions environnementales et l'organisation des services de soins » (Gouvernement du Québec, 1988, p. 63). L'environnement social, économique et culturel aurait donc une influence sur la santé ou la maladie des personnes. La complexité de l'interaction entre l'homme et son environnement ainsi que l'impact de la maladie sur l'individu et sur son milieu de vie font partie du savoir du praticien social. Ce dernier connaît la signification psychosociale de la maladie, les réactions émotives et les comportements qu'elle peut déclencher, ainsi que ses conséquences sur la vie de la personne et sur celle de ses proches, notamment le changement temporaire ou permanent dans l'accomplissement des différents rôles sociaux.

\section{Le diagnostic social et l'intervention psychosociale}

L'expertise des systèmes sociaux chez le praticien social peut se comparer à l'expertise du système nerveux chez le médecin. Le diagnostic social et l'intervention psychosociale permettent de favoriser la prise en charge par la personne de son rétablissement, la mobilisation positive de son environnement, 
l'adaptation des ressources du milieu de vie de la personne et la sollicitation des services communautaires appropriés.

Le praticien social intervient avec une attitude qui valorise l'estime et l'affirmation de soi. Cette approche favorise la résolution de problèmes liés à la maladie ou l'inverse, c'est-à-dire la résolution de problèmes sociaux qui affectent la santé. Le praticien social contribuera au virage ambulatoire en faisant en sorte que le «social» ne soit pas le facteur qui exacerbe le «biologique» et vice versa.

Les praticiens sociaux ont traditionnellement «joué un rôle charnière important entre l'hôpital et la communauté ». Leurs habiletés

- dans l'analyse et la compréhension des dynamiques relationnelles et systémiques (individus-environnement),

- dans la connaissance et l'utilisation de la communauté et de ses ressources,

- dans l'art d'organiser et de coordonner des services pour les personnes,

- dans la représentation des intérêts et des droits des clientèles,

- dans l'impact des limitations physiques et sociales sur l'organisation du quotidien,

- dans la négociation et la médiation ainsi que dans la liaison des services et collaborations entre les différentes instances en présence (Gagnon-Hotte, 1995, p. 11) font d'eux des acteurs de choix pour réaliser harmonieusement le transfert d'une partie des soins vers le milieu de vie des personnes.

\section{Le rôle de représentation}

Le praticien social en milieu hospitalier connaît la culture hospitalière et il est formé au rôle d'advocacy. Habilité à faire les liens entre les différentes composantes du système de la personne, il peut ainsi faire les représentations nécessaires, afin de s'assurer que l'organisation des services externes complémentaires correspond à leurs besoins pour éviter notamment un retour à l'hôpital. 


\section{LES RÉPERCUSSIONS SUR LES CLIENTÈLES}

La médecine et la chirurgie de jour, la diminution des durées de séjour en milieu hospitalier supposent nécessairement un engagement différent de l'usager et de son entourage dans la dispensation des soins de santé. Ce qui était assumé jusqu'à présent par l'hôpital est maintenant restreint à la période intensive de traitement et tout l'aspect intermédiaire de soins et services est transféré au réseau communautaire, à l'individu et à son entourage.

Cela modifie de façon substantielle les relations de l'usager avec son environnement familial et social. Depuis plus de vingtcinq ans, les familles avaient modifié leur lien d'interdépendance et transféré en bonne partie à l'État-providence la mission du maintien à domicile, de la convalescence et des soins. Cela faisait partie des responsabilités dévolues à l'État. Tel que démontré, le virage ambulatoire suppose une redéfinition des responsabilités de l'hôpital, des CLSC, des organismes communautaires, mais il génère aussi une renégociation des dynamiques familiales et intergénérationnelles.

\section{Les aidants naturels}

Ramener la diminution des durées de séjour et la médecine ambulatoire à une vision médicale seulement des soins, c'est nier tout l'aspect soutien, surveillance, activités domestiques, réorganisation, encadrement que les personnes nécessitent lorsqu'elles retournent à domicile plus tôt et avec des besoins plus importants ou aux prises avec un diagnostic sévère ayant un impact émotionnel certain sur elles et sur leurs proches.

Parler des aidants naturels à l'aube de l'an 2000, c'est encore et surtout parler des aidantes naturelles. Les exigences du virage ambulatoire auront donc principalement un impact sur les conditions de vie des femmes qui se retrouveront vraisemblablement avec des charges de soutien ou de planification de soutien alourdies.

\section{Les dynamiques relationnelles}

Les praticiens de service social en milieu de santé sont et seront au cœur de la préparation des retours à domicile lourds et de la réorganisation que cela nécessite. Leur expertise auprès de la 
famille les prépare à inciter et à aider celle-ci à se resolidariser par rapport à son proche hospitalisé. «... il est de la plus grande importance qu'une attention particulière soit portée aux personnes et aux familles qui vivent de tels changements, afin qu'elles soient respectées, comprises, soutenues dans leur démarche et ce, autant dans leur ambivalence et dans leur résistance que dans leur détresse et leur décision» (Gagnon-Hotte, 1995, p. 6).

Penser que les rapports intergénérationnels seront peu importants dans ces révisions déchirantes des répartions de charges, de biens et services, c'est se prêter à un nouvel aveuglement après celui de la négation des tensions actuelles entre les générations.

\section{Jacques Grand'Maison}

Si le soutien est bien planifié, si les capacités des aidants familiaux sont bien évaluées, mesurées et respectées, le virage peut aussi avoir des effets positifs sur l'usager et son entourage. En effet, l'individu devient partenaire actif dans la prise en charge de sa santé: on l'informe, l'implique, lui enseigne à prendre soin de lui (empowerment). De plus, la dynamique familiale se trouve bouleversée et déstabilisée par ce virage: les nouvelles réalités exigent que l'on passe d'une notion individuelle, où chacun a des contacts dissociés et indépendants avec les autres membres de la famille, à une notion de soutien mutuel et d'interdépendance. Si le partage est bien équilibré, si les familles sont soutenues et accompagnées, celles-ci peuvent et devraient y gagner sur certains plans, telles la solidarité et la sécurité affective. Jacques Grand'Maison, dans sa recherche sur les familles et les liens intergénérationnels, ne soutient-il pas qu'une des grandes pertes de notre ère individualiste, $c^{\prime}$ est l'absence de projets et d'interdépendance entre les générations (Grand-Maison, 1994).

\section{Les coûts associés prévisibles}

Deux derniers points qu'il est non négligeable de mentionner, même si ce ne sont que des hypothèses pour le moment, sont l'impact économique et l'impact sur la qualité de vie et les libertés individuelles qu'aura la modification du mode de dispensation des soins de santé.

Cet impact économique peut se traduire par des coûts reliés aux évaluations en préhospitalisation, aux transports multiples 
pour les soins en externe au CLSC, à la clinique, à l'hôpital et à l'hôtellerie souvent nécessaire pour les gens venant des périphéries. Peut s'ajouter l'aide qui serait requise pour la convalescence. Le citoyen, déjà lourdement taxé, paiera le même impôt pour ses services de santé, mais subira une taxe spéciale à l'utilisateur, indirecte mais réelle, en assumant certains frais découlant des nouveaux modes de pratique. C'est un aspect auquel le praticien social doit être sensibilisé puisqu'il risque d'affecter directement l'usager.

Nonobstant les possibilités de resserrements familiaux, il faut maintenant comprendre que virage ambulatoire voudra dire, pour les parents, conjoints, enfants et proches des personnes malades, une attribution plus importante du temps familial et personnel à consacrer à celles-ci. À moins de bénéficier de substituts à la maison, les aidants naturels devront souvent consacrer une partie de leurs congés, périodes de repos ou loisirs personnels aux soins ou à la convalescence de leur proche dans le besoin. Il serait illusoire de compter uniquement sur le réseau communautaire et de services, dans une période où l'État cherche à se désengager de certaines de ses charges financières et où l'accessibilité aux ressources de maintien à domicile tient désormais compte des revenus individuels.

Ces changements majeurs dans le mode de dispensation des soins et services de santé nécessiteront une période de transition, plus ou moins longue, nécessaire à l'évolution des mentalités et des habitudes. Les praticiens sociaux ont un rôle important à jouer auprès des gens pour expliquer, soutenir et favoriser ce changement. Ils connaissent bien et croient aux capacités des personnes à se prendre en charge, à s'adapter aux situations nouvelles et à devenir proactives dans leur vie. Parallèlement, ils sont habilités à évaluer la vulnérabilité des personnes. Leur grande responsabilité portera sur la justesse de cette évaluation, sur la transmission de cette opinion professionnelle et de ses implications à l'hôpital, à l'usager, à ses proches, de même qu'aux acteurs du réseau qui contribueront à l'approche horizontale de service.

\section{LES DÉFIS DES PROCHAINES ANNÉES}

Les défis qui attendent les services sociaux hospitaliers sont importants, à la taille des changements qui surviennent actuellement dans le réseau. Ceux qu'il est possible d'identifier vont solliciter une fois de plus leurs capacités d'adaptation. 


\section{Le virage ambulatoire et l'approche clinique}

Plusieurs problèmes de santé, ainsi qu'il a été mentionné, seront désormais traités en externe ou en court séjour et l'hospitalisation de plusieurs usagers, en médecine, en chirurgie et en psychiatrie, sera abrégée. Sur le plan professionnel, le premier défi du service social consistera à développer encore davantage des modèles d'intervention où l'évaluation allie à la fois la brièveté dans le temps et la précision dans la saisie de l'état émotif et social du système-client, tout en permettant d'établir rapidement la relation avec lui.

Il sera utile sans doute que l'une des prochaines démarches de perfectionnement à privilégier vise à rafraîchir et à approfondir ces réalités. Plus encore, un important travail de recherche théorique et clinique devrait être entrepris pour aider à confirmer les types d'approches brèves les plus pertinentes et efficaces pour le travail du praticien social en milieu de santé.

En outre, le développement d'outils mesurant l'apport de l'intervention psychosociale à l'amélioration de la santé et à l'équilibre des coûts du système de santé devra être accéléré à des fins de priorisation de ressources et d'investissement professionnel mieux ciblé.

Pour un ensemble de situations, le travail du service social consistera en une évaluation de la situation psychosociale et à l'organisation d'un plan d'intervention dont une grande partie sera réalisée en collaboration avec les organismes du milieu, les CLSC en particulier. Actuellement, la communication, la transmission de l'information et la poursuite du plan d'intervention sont souvent l'objet d'un processus lent et lourd entre le centre hospitalier et le CLSC. Ce processus devra être allégé et demandera un changement dans les façons mutuelles de faire. La nouvelle situation commandera en effet que les usagers transitent d'un établissement à l'autre avec plus de fluidité et moins de barrières. Les praticiens sociaux ont un rôle charnière dans la transmission aux intervenants du CLSC des réalités sociales et émotives vécues par le client, ces dernières pouvant faire la différence entre une récupération réussie ou un état de santé qui périclite.

De même, il est important que les praticiens sociaux des CLSC puissent faire le point avec le service social hospitalier lors de l'hospitalisation d'une personne auprès de laquelle ils interviennent. Les futures ententes entre ces deux établissements doivent inclure la préoccupation de cette réalité. Aussi est-il 
important que le service social participe à l'élaboration des nouveaux modes de collaboration et soit présent dans les comités de concertation centre hospitalier / CLSC qui se mettent actuellement en place dans la plupart des milieux.

Les modes actuels de transaction avec les ressources d'hébergement public, particulièrement en ce qui concerne le dépannage et le répit, auraient aussi avantage à être simplifiés pour assurer une accessibilité plus grande et plus facile.

Le secteur des ressources privées dans le domaine de la santé se développe rapidement : services de maintien à domicile, maisons d'hébergement, de dépannage, de convalescence. Le virage ambulatoire invite à une plus grande collaboration avec ces ressources. C'est pourquoi il est important que les institutions en systématisent les normes d'utilisation et les modes de contrôle de la qualité étant donné qu'en matière de santé les ressources ne doivent pas être laissées aux seules règles du marché et de la libre entreprise. Le but ici n'est pas de bouder ces ressources, mais de voir à protéger des clientèles vulnérables et, plus globalement, de participer à la définition des services qui doivent s'y retrouver et à leur qualité.

\section{Les clientèles lourdes}

S'il abrège le séjour de certaines catégories de personnes, le virage ambulatoire aura aussi comme effet la concentration dans les centres hospitaliers d'usagers aux prises avec des maladies graves ou évolutives ou présentant un diagnostic réservé (cancer, diabète, traumatisme, maladie mentale complexe, sida, maladie cardiaque ou rénale).

La sévérité de leurs problèmes de santé exige en effet qu'ils reçoivent leurs services du centre hospitalier, à cause de l'important plateau technique nécessaire. La complexité de ces maladies requiert aussi la présence d'une équipe multidisciplinaire spécialisée, relevant d'un même établissement. Le service social devra être présent dans l'ensemble de ces équipes dans un effort pour articuler un plan de traitement global qui soit sensible à l'ensemble des besoins de la personne, à sa réassurance et à celle de ses proches.

Or, ces équipes sont encore peu nombreuses et le discours sur la réforme du système de santé ne semble pas en faire un instrument d'intervention, puisqu'on parle plutôt de déprofessionnalisation de l'intervention. Le défi va consister ici à être 
actif dans le processus de la réforme dans les différents centres hospitaliers et à faire valoir que le développement des équipes multidisciplinaires est un outil important, non seulement pour une prise en charge globale de l'usager, mais aussi comme apport à l'efficacité du système de santé.

\section{EN CONCLUSION \\ La nécessaire préoccupation des réalités sociales et émotives}

La réorganisation du système de santé va donc amener l'usager à consommer les services de santé de façon différente et dans des institutions diversifiées; elle va également solliciter les intervenants à s'adapter à de nouveaux modèles. Un fait va demeurer cependant: les réalités émotives et sociales vont continuer à être parties intégrantes du retour à la santé ou de la nécessaire adaptation à la maladie. Oublier cela, c'est risquer une évolution partielle ou négative de plusieurs usagers, et imposer un coût social important à ceux que la maladie déstabilise déjà ; or, les coûts sociaux se traduisent rapidement par des coûts financiers.

Les praticiens sociaux en milieu de santé adhèrent aux préoccupations actuelles d'une gestion efficace et d'une diminution des coûts du système de santé; ils sont conscients qu'il s'agit là d'une condition de sa survie.

Cette nécessité ne doit pas occulter une autre réalité : la diminution des coûts ne pourra s'effectuer qu'en continuant simultanément à améliorer les facteurs qui ont un impact important sur la santé. Ces facteurs, nous les avons cités d'entrée de jeu, s'appellent sentiment de contrôle sur sa vie et capacité de faire face aux difficultés. Ces déterminants de la santé individuelle sont liés à la prospérité générale d'une société donnée, mais aussi à la solidité du tissu social assurée par le soutien qu'on peut trouver dans cette communauté, par la capacité de s'y développer et l'invitation à participer au projet commun. Investir dans ces réalités, c'est contribuer à maintenir et à améliorer la santé d'une société.

Au niveau des individus, il demeure tout aussi important de continuer à intervenir auprès d'eux, afin qu'ils utilisent au mieux ces outils pour s'adapter aux conséquences sociales et émotives de la maladie et pour améliorer ou regagner leur état de santé. 
Il est en effet essentiel que notre société, même en des temps difficiles, continue à adhérer à la définition énoncée par l'Organisation mondiale de la santé: la santé est un état de bien-être physique, psychologique et social. C'est là un investissement qui rapportera à court, moyen et long terme ; les difficultés actuelles ne doivent pas le faire oublier.

\section{Références bibliographiques}

DESROSIERS, Gilles (1995). Les services médicaux de base dans le milieu, Document de travail, Sherbrooke: Régie régionale de la santé et des services sociaux de l'Estrie, non publié, $15 \mathrm{p}$.

Document préparé pour la conférence des sous-ministres de la santé et des services sociaux des gouvernements fédéral, provinciaux et des territoires, Faire mieux avec moins pour une utilisation efficace des hôpitaux canadiens, juin 1994.

FÉDÉRATION DES CLSC (1995). Virage ambulatoire. Introduction générale, juin, $12 \mathrm{p}$.

GAGNON-HotTE, L. (1995). Mémoire présenté par les services sociaux hospitaliers dans le cadre de la consultation sur l'organisation des services, Sherbrooke, mai, non publié, $11 \mathrm{p}$.

GOUVERNEMENT DU QUÉBEC (1988). Rapport de la commission d'enquête sur les services de santé et les services sociaux, $803 \mathrm{p}$.

Grand'Maison, Jacques et Solange Lefebvre (1994). La Part des Aînés, Recherche-Action, Quatrième dossier, Éditions Fides, 362 p.

HÉBERT, L. et H. OUELLET (1995). L'Hôpital de demain, notes de conférence, juin.

Ministère de la SANTÉ et Des Services Sociaux (1993). Plan de répartition de l'effectif médical au Québec - 1993-1996, Québec: MSSS, Direction générale des relations professionnelles, $149 \mathrm{p}$.

RÉGIE RÉGIONALE DE LA SANTÉ ET DES SERVICES SOCIAUX DE QUÉBEC (1995). Centres de services ambulatoires, Cadre de référence sur l'organisation des services, juin, non publié, $27 \mathrm{p}$.

RÉGIE RÉGIONALE DE LA SANTÉ ET DES SERVICES SOCIAUX DE QUÉBEC (1995). Plan triennal de transformation du système régional de santé et de services sociaux, Québec, juin, $79 \mathrm{p}$.

RENAUD, M. et L. BOUCHARD (1994). «Expliquer l'inexpliqué: l'environnement social comme facteur clé de la santé», Interface. Revue de recherche, vol. $15, \mathrm{n}^{\circ} 2$, mars-avril.

Rochon, Jean (1995). Allocution, $31^{e}$ Congrès de l'Association des hôpitaux du Québec. 